\title{
Continuous Digital Assessment for Weight Loss Surgery Patients
}

\author{
Ernesto Ramirez $^{a} \quad$ Nikki Marinsek ${ }^{a} \quad$ Benjamin Bradshaw ${ }^{a}$ \\ Robert Kanard $^{\mathrm{b}}$ Luca Foschini ${ }^{\mathrm{a}}$ \\ a Evidation Health, San Mateo, CA, USA; ${ }^{\text {b} S a n t a ~ B a r b a r a ~ C o t t a g e ~ H o s p i t a l, ~}$ \\ Santa Barbara, CA, USA
}

\section{Keywords}

Wearables $\cdot$ Surgery $\cdot$ Remote monitoring $\cdot$ Real-world data

\section{Abstract}

We conducted a survey about recent surgical procedures on a large connected population and requested each individual's permission to access data from commercial wearable devices they may have been wearing around the time of the procedure. For subcohorts of 66-118 patients who reported having a weight loss procedure and who had dense Fitbit data around their procedure date, we examined several daily measures of behavior and physiology in the 12 weeks leading up to and the 12 weeks following their procedures. We found that the weeks following weight loss operations were associated with fewer daily total steps, smaller proportions of the day spent walking, lower resting and 95th percentile heart rates, more total sleep time, and greater sleep efficiency. We demonstrate that consumer-grade activity trackers can capture behavioral and physiological changes resulting from weight loss surgery and these devices have the potential to be used to develop measures of patients' postoperative recovery that are convenient, sensitive, scalable, individualized, and continuous.

(C) 2020 The Author(s)

Published by S. Karger AG, Basel

\section{Introduction}

Postoperative follow-up for patients undergoing weight loss surgical procedures is typically episodic, with in-person assessments completed at 3-6 months, and then yearly thereafter [1]. Moreover, much of the existing literature describes short observations (approx. 1 week), commonly using accelerometer-based measures of physical activity, during the 6- to 12-month postoperative period [2,3]. While there has been some interest in using commercial wearable devices to describe postoperative behavior, the currently 
Ramirez et al.: Continuous Digital Assessment for Surgery Patients

available research has been limited in scope, typically bound to short observation windows and/or only focused on assessing daily physical activity behavior in the form of daily total steps [4-9]. Our current understanding of the changes in postoperative behavior and physiology may not reflect the true relationship between these changes and outcomes of interest such as successful weight loss, adverse event incidence, and resolution of obesity-related medical comorbidities.

Data from new consumer and medical devices may be able to bridge this gap by providing much-needed insights about the trajectory of physical functioning after weight loss surgery on a finer timescale and longer time course [10]. As a first step towards understanding the potential for new devices and data streams, we completed a preliminary retrospective assessment of the impact of weight loss surgery on numerous behavioral and physiological features derived from Fitbit consumer activity tracker devices.

\section{Methods}

Data Collection

Evidation Health currently supports a mobile consumer application called Achievement that rewards members based on completing health-related behaviors and participating in research. Achievement members can connect activity trackers and health applications and authorize Achievement to automatically and continuously ingest connected data streams. We deployed a short survey to Achievement members that asked about medical procedures they have undergone in the 2 years prior to taking the survey.

\section{Data Processing}

A total of 50,938 individuals reported undergoing a medical procedure, and 1,203 of those individuals reported undergoing a weight loss procedure. With the participants' permission, we linked their survey responses to their activity data from June 1,2015 , to January 1, 2019. Only participants with connected Fitbit device data $(n=675)$ were used in the analysis. Fitbit devices have been found to be valid and reliable for capturing step, heart rate, and sleep data [11-14]. We restricted the analysis to the time period encompassing 12 weeks (84 days) before to 12 weeks after the participants' reported procedure dates $(n=$ 520 ). We then applied a data density requirement (defined as having no more than 7 consecutive days of missing data within the 24 -week observation window) to filter out participants with sparse and/or inconsistent device data. Finally, we linearly interpolated gaps in the data. The final analysis set consisted of 118,66, and 87 patients with dense step, heart rate, and sleep features, respectively. The data processing process is illustrated in Figure 1.

\section{Statistical Analysis}

We focused on examining the impact of weight loss procedures on 6 daily behavioral features, 3 of which (total sleep time, sleep efficiency, and resting heart rate) were accessible from the public Fitbit application programming interface (https://dev.fitbit.com/build/ reference/web-api/) and 3 of which were manually derived from the minute-level intraday activity data (total step count, fraction of minutes per day with $>0$ steps, and 95 th percentile heart rate) [15-20]. To reduce the impact of day-of-the-week effects, we computed the weekly mean of each feature for each participant for each week of the observation window (online suppl. Tables 1-3; see www.karger.com/doi/10.1159/000506417 for all online suppl. material). Finally, we ran a series of Wilcoxon signed-rank tests to determine if the distribution of the weekly feature means was significantly different than the distribution of weekly means from a baseline week ( 12 weeks prior to the operation). We chose week 12 prior to the 


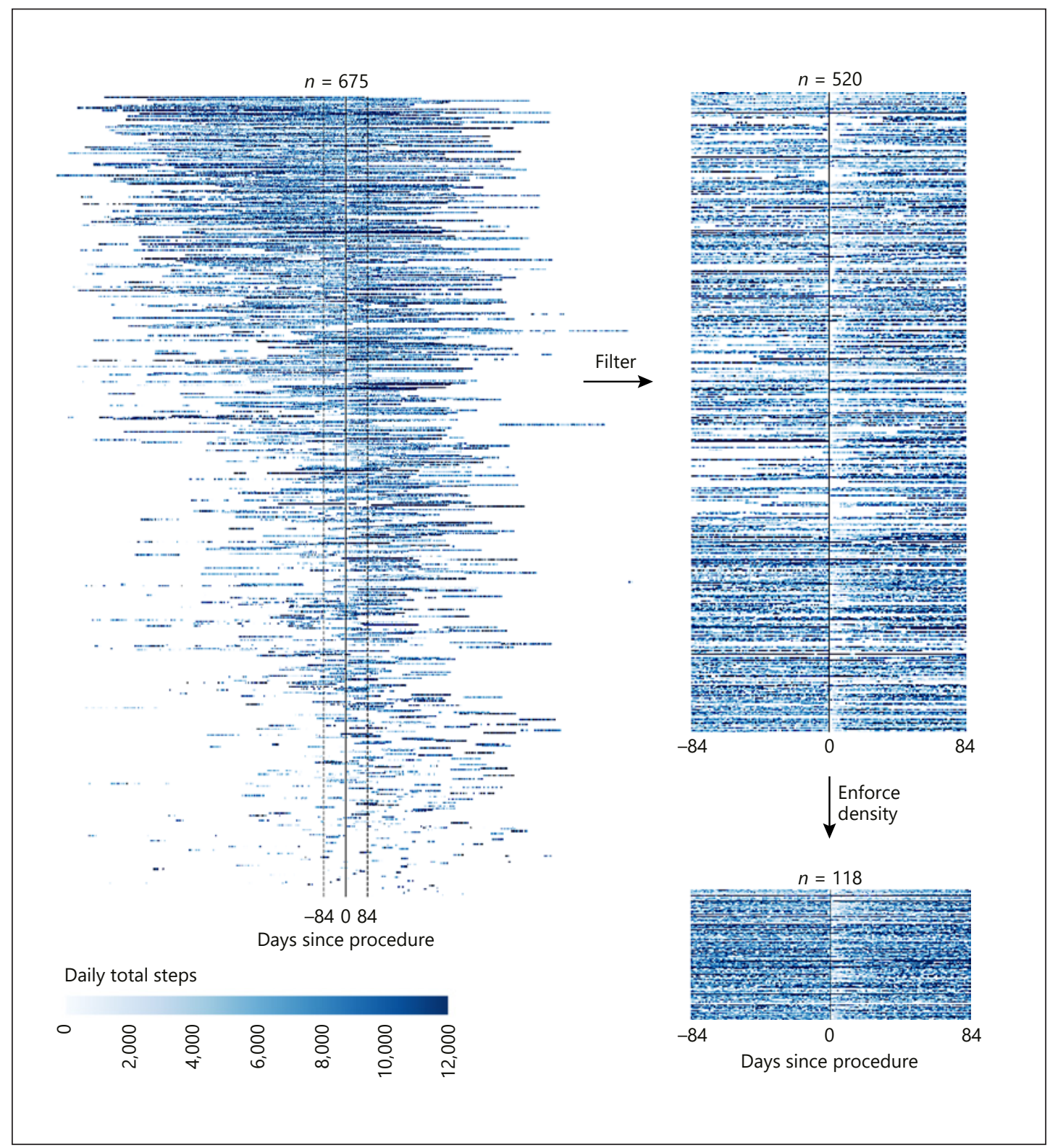

Fig. 1. Sample data reduction process for physical activity data (steps) included in the analysis. We began data processing by exploring (a) the representation of the availability of daily step data for the sample of 675 participants with activity data; then we (b) reduced the sample of participants to include only those with daily step data for the 84-day period before and after the procedure and (c) further reduced the sample to include only those participants who met the "dense data" requirements of at most 7 consecutive days of missing data within 84 days of the procedure date.

operation as the baseline week in order to account for potential behavioral changes in the weeks leading up to surgery associated with surgical center recommendations or mandates instituted by insurance providers [21]. We report several significant thresholds that correspond to (a) no adjustment ( $p<0.05$ ), (b) a Bonferroni adjustment for running 23 tests per feature $(p<0.00217)$, and (c) a Bonferroni adjustment for running 138 total tests ( 23 weeks $\times$ 6 features; $p<0.00036$ ). 
Ramirez et al.: Continuous Digital Assessment for Surgery Patients

Table 1. Participant demographics

\begin{tabular}{lllll}
$\begin{array}{l}\text { Reported a weight } \\
\text { loss procedure }\end{array}$ & $\begin{array}{l}\text { Had activity data } \\
\text { from Fitbit }\end{array}$ & Analysis set & \\
\cline { 3 - 5 }$(n=1,203)$ & $(n=675)$ & steps & heart rate & sleep \\
& & $(n=118)$ & $(n=66)$ & $(n=87)$
\end{tabular}

Gender

\begin{tabular}{|c|c|c|c|c|c|}
\hline Female & $1,047(87 \%)$ & $616(91 \%)$ & $109(92 \%)$ & $60(91 \%)$ & $81(93 \%)$ \\
\hline Male & $133(11 \%)$ & $49(7 \%)$ & $7(6 \%)$ & $5(8 \%)$ & $5(6 \%)$ \\
\hline Other & $3(<1 \%)$ & $2(<1 \%)$ & $0(0 \%)$ & $0(0 \%)$ & $0(0 \%)$ \\
\hline $\begin{array}{l}\text { Unavailable } \\
e^{1}\end{array}$ & $20(2 \%)$ & $8(1 \%)$ & $2(2 \%)$ & $1(1 \%)$ & $1(1 \%)$ \\
\hline Mean age $\pm S D$, years & $36.0 \pm 10.2$ & $36.4 \pm 9.5$ & $38.0 \pm 9.8$ & $37.1 \pm 8.8$ & $37.3 \pm 9.5$ \\
\hline $\begin{array}{l}\text { Unavailable } \\
\text { ucation }\end{array}$ & $17(1 \%)$ & $8(1 \%)$ & $1(<1 \%)$ & $1(1 \%)$ & $1(1 \%)$ \\
\hline Doctorate, MD & $10(<1 \%)$ & $4(<1 \%)$ & $0(0 \%)$ & $0(0 \%)$ & $0(0 \%)$ \\
\hline Graduate degree & $166(14 \%)$ & $89(13 \%)$ & $16(14 \%)$ & $12(18 \%)$ & $9(10 \%)$ \\
\hline College degree (AS or BS) & $477(40 \%)$ & $295(44 \%)$ & $59(50 \%)$ & $32(48 \%)$ & $46(53 \%)$ \\
\hline Some college & $272(23 \%)$ & $151(22 \%)$ & $20(17 \%)$ & $12(18 \%)$ & $18(21 \%)$ \\
\hline Trade or vocational training & $91(8 \%)$ & $54(8 \%)$ & $5(4 \%)$ & $2(3 \%)$ & $3(3 \%)$ \\
\hline High school diploma/GED & $104(9 \%)$ & $51(8 \%)$ & $14(12 \%)$ & $6(9 \%)$ & $9(10 \%)$ \\
\hline No high school diploma & $18(1 \%)$ & $9(1 \%)$ & $0(0 \%)$ & $1(2 \%)$ & $0(0 \%)$ \\
\hline Unavailable & $65(5 \%)$ & $22(3 \%)$ & $4(3 \%)$ & $1(2 \%)$ & $2(2 \%)$ \\
\hline
\end{tabular}

GED, general equivalency diploma. ${ }^{1}$ Age at the time of the procedure is estimated based on information from a patient ID-linked survey completed at a different time point than the medical event survey.

\section{Results}

Patients included in the final analysis sample overwhelmingly were female and college educated, with a mean age under 40 years. The full demographic characteristics available for this analysis are presented in Table 1.

The changes in patients' steps, heart rate, and sleep in the 12 weeks leading up to and the 12 weeks following their weight loss procedures are illustrated in Figure 2. Each of the 6 features we examined was significantly different from that at baseline (defined as preoperative week 12) in at least 1 of the weeks following the operation. The weekly means for each feature are reported in the online supplementary materials (Tables 1-3).

The patients were generally less physically active following their weight loss procedures (Fig. 2). In week 0 (which consisted of the operation day and the following 6 days), the total daily steps decreased by an average of 2,720 steps per day, from $7,030( \pm 3,438)$ steps per day in preoperative week 12 to $4,310( \pm 3,620)$ steps per day (Wilcoxon test, $p<0.0001)$. The weekly average total step counts remained significantly lower than those at baseline up until postoperative week 4. Similarly, the patients spent greater proportions of the day not taking any steps in the weeks following their operations. The average fraction of minutes per day with at least 1 step decreased by $39 \%$ in the week following the procedure (from $0.17[ \pm 0.07]$ on preoperative week 12 to 0.11 [ \pm 0.07 ] on week 0 ), and remained significantly lower than at baseline for a total of 4 weeks. Interestingly, the average weekly total step counts surpassed the baseline levels in the third month following the procedure, but these differences were only significant without adjusting for multiple comparisons.

Both resting heart rate and upper bounds (95th percentile) of daily heart rate decreased in the 12-week period following the weight loss procedures. The decreases in resting heart rate were especially pronounced, with the average weekly resting heart rates decreasing by 


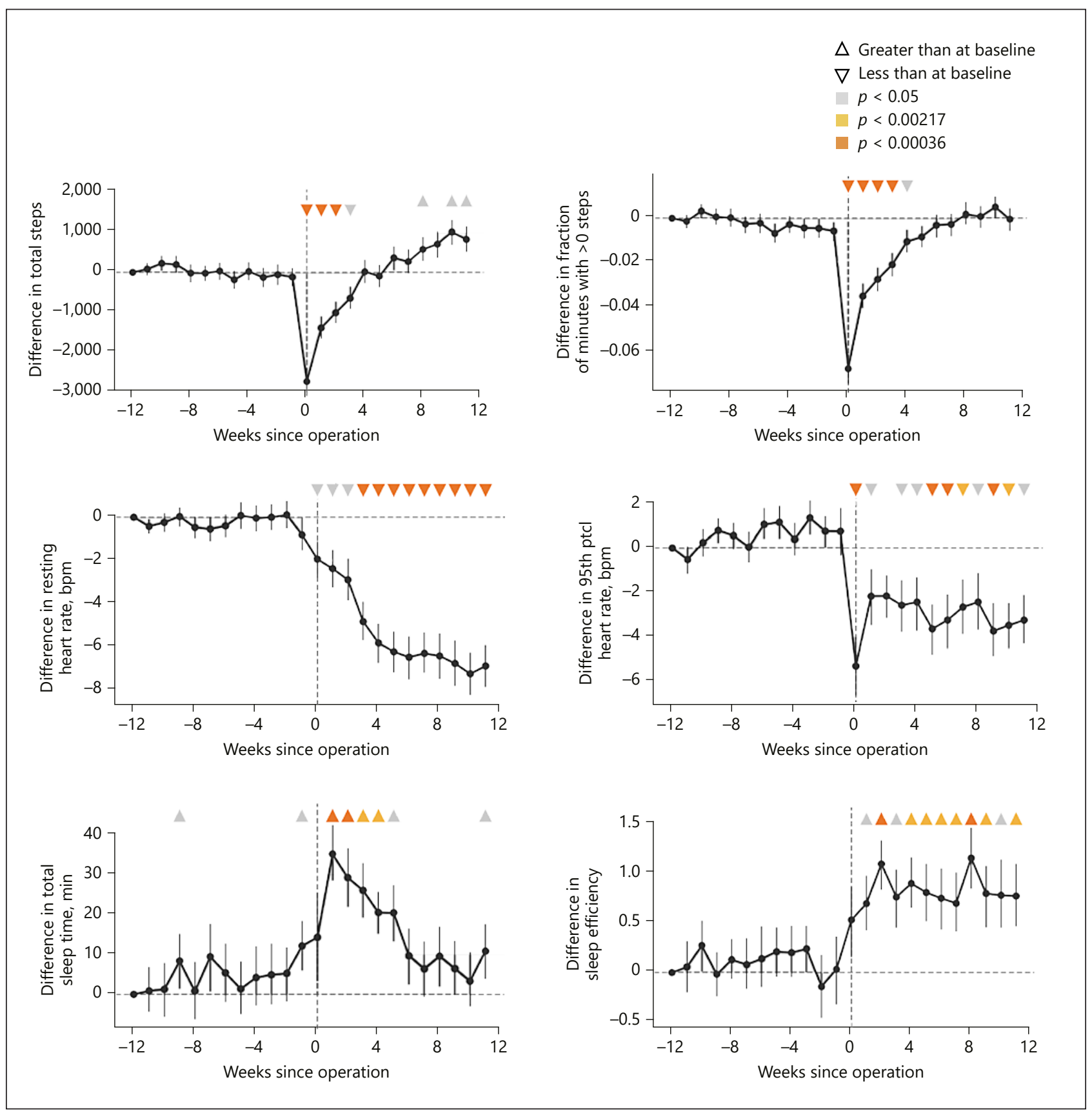

Fig. 2. Changes in activity features throughout the pre- and postoperative period. Differences in weekly feature means relative to the feature mean of a baseline week (12 weeks prior to the operation) across patients. Error bars correspond to the standard deviation of differences across patients. Weeks that are significantly different from baseline are denoted with triangles, with colors that correspond to different significance levels (gray: $p<0.05$; yellow: $p<0.00217$; orange: $p<0.00036$ ), and directionality indicating an increase or a decrease relative to baseline.

at least 6 beats per minute (bpm) compared to baseline from postoperative week 5 onward. Similarly, the 95th percentile heart rates decreased by approximately 2-4 bpm compared to baseline from postoperative week 2 onward, but only some of the postoperative weeks were significantly different from the baseline after adjusting for multiple comparisons. 
Ramirez et al.: Continuous Digital Assessment for Surgery Patients

We observed short-term increases in total sleep time in the first several weeks following patients' operations and longer-term increases in sleep efficiency. On average, the participants slept $14 \mathrm{~min}$ more than at baseline in the week following their operation (week $0 ; p>$ $0.05)$ and a peak of 35 min more in the second postoperative week $(p<0.001)$. Sleep efficiency increased slightly following the weight loss procedures, increasing from 91.0 in preoperative week 12 to a maximum of 92.1 in postoperative week $2(p<0.0001)$.

None of the weekly feature means were significantly different than those at baseline in the preoperative period after adjusting for multiple comparisons.

\section{Discussion}

This is the first examination of changes in continuous behavioral and physiological measures after a weight loss procedure that makes use of data derived from consumer wearable devices. In addition to exploring the trajectory of change of specific and meaningful components of physical functioning and physiology, we found support that trajectory profiles demonstrate differences in behavior that may be missed if only episodic measures were taken during infrequent clinical encounters.

We observed a variety of changes inferred from consumer wearable device data that have potential for providing additional insight into what occurs during the postoperative and recovery periods for individuals who undergo a weight loss procedure. For example, we found the resting heart rate to be decreased on average by $3 \mathrm{bpm}$ the first week after the procedure and by $7 \mathrm{bpm}$ after 12 weeks. These insights may be useful as clinicians and researchers explore methods of improving outcomes that are more relevant to patients and reduce adverse events. The common marker of success for weight loss procedures is weight reduction; however, postoperative outcomes related to quality of life have been found to be more important to patients when compared to health professionals [22].

The current study design is based on individuals' self-reported medical procedure information. While such procedures tend to be impactful and recall should be high, there is a potential for recall bias, especially surrounding the procedure date. Approximately $46 \%$ of the individuals with dense data included in this analysis reported undergoing a procedure within 1 year (365 days) of their survey completion date, hopefully reducing errors in reporting the procedure date. Additionally, the medical procedure survey was designed to assess a variety of procedures, and information about specific surgery types was not available.

One of the common criticisms of wearable devices is the lack of understanding regarding device wear time in free-living conditions. We found that approximately $5-10 \%$ of the individuals in our connected population who self-reported having a weight loss procedure met the data density requirements for inclusion in this analysis. While this may appear low, individuals were not purposefully recruited to use an activity tracking device during their procedures, the data density thresholds were relatively strict, and this analysis was limited to only a single device manufacturer (Fitbit). Additionally, survey responders were able to reference surgical dates that were prior to the date they joined Achievement and/or purchased a Fitbit device.

Prospective studies with individuals who are planning to undergo a weight loss procedure may provide additional support for the use of consumer devices to continuously assess behavioral and physiological changes and their relationship to outcomes of interest. For example, a recent prospective study of 143 patients who underwent common elective surgical procedures found significant differences in daily step counts between the preoperative baseline, 1-week-postoperative, and 4-week-postoperative phases, as well as differences between surgery types [9]. While this analysis focused on comparing weekly feature means, features 
of connected wearable devices and the data they collect have the potential be used to develop day-by-day recovery profiles similar to growth curves used for infants and children [23]. These recovery profile curves could be used to remotely monitor patients postoperatively and identify when recovery deviates from a standard trajectory derived from real-world data collected in prospective clinical trials.

\section{Acknowledgements}

The authors are grateful to the participants, members of the Evidation-developed Achievement Platform, for their participation in this research endeavor. The authors also thank Steven Shi for helpful editing and manuscript review.

\section{Statement of Ethics}

This study received expedited review and IRB approval from Solutions IRB (Protocol ID \#2019/04/14). Waiver of informed consent was granted by the IRB due to the retrospective design of the study (see online suppl. materials).

\section{Disclosure Statement}

E.R., N.M., and B.B. are employees of Evidation Health; L.F. is a cofounder and employee of Evidation Health.

\section{Funding Sources}

This work was self-funded.

\section{Author Contributions}

E.R., N.M., B.B., and L.F. contributed to the study design, result interpretation, and paper revisions; N.M. conducted the data cleaning and data analysis with guidance from E.R. and L.F.; E.R. and N.M. drafted the paper; R.K. provided clinical interpretations and provided editorial feedback. All authors approved the completed version.

\section{References}

1 Bond DS, Jakicic JM, Unick JL, Vithiananthan S, Pohl D, Roye GD, et al. Pre- to postoperative physical activity changes in bariatric surgery patients: self report vs. objective measures. Obesity (Silver Spring). 2010 Dec; 18(12):2395-7.

2 King WC, Chen JY, Belle SH, Courcoulas AP, Dakin GF, Elder KA, et al. Change in pain and physical function following bariatric surgery for severe obesity. JAMA. 2016 Apr;315(13):1362-71.

3 Herring LY, Stevinson C, Davies MJ, Biddle SJ, Sutton C, Bowrey D, et al. Changes in physical activity behaviour and physical function after bariatric surgery: a systematic review and meta-analysis. Obes Rev. 2016 Mar; 17(3):250-61.

4 Mobbs RJ, Phan K, Maharaj M, Rao PJ. Physical activity measured with accelerometer and self-rated disability in lumbar spine surgery: a prospective study. Global Spine J. 2016 Aug;6(5):459-64. 
Ramirez et al.: Continuous Digital Assessment for Surgery Patients

5 Low CA, Bovbjerg DH, Ahrendt S, Choudry MH, Holtzman M, Jones HL, et al. Fitbit step counts during inpatient recovery from cancer surgery as a predictor of readmission. Ann Behav Med. 2018 Jan;52(1):88-92.

6 Cook DJ, Thompson JE, Prinsen SK, Dearani JA, Deschamps C. Functional recovery in the elderly after major surgery: assessment of mobility recovery using wireless technology. Ann Thorac Surg. 2013 Sep;96(3):105761.

7 Symer MM, Abelson JS, Milsom J, McClure B, Yeo HL. A mobile health application to track patients after gastrointestinal surgery: results from a pilot study. J Gastrointest Surg. 2017 Sep;21(9):1500-5.

8 Toogood PA, Abdel MP, Spear JA, Cook SM, Cook DJ, Taunton MJ. The monitoring of activity at home after total hip arthroplasty. Bone Joint J. 2016 Nov;98-B(11):1450-4.

9 Carmichael H, Overbey DM, Hosokawa P, Goode CM, Jones TS, Barnett CC Jr, et al. Wearable technology - a pilot study to define "normal" postoperative recovery trajectories. J Surg Res. 2019 Dec;244:368-73.

10 Bond DS, Thomas JG. Measurement and intervention on physical activity and sedentary behaviours in bariatric surgery patients: emphasis on mobile technology. Eur Eat Disord Rev. 2015 Nov;23(6):470-8.

11 Appelboom G, Taylor BE, Bruce E, Bassile CC, Malakidis C, Yang A, et al. Mobile phone-connected wearable motion sensors to assess postoperative mobilization. JMIR Mhealth Uhealth. 2015 Jul;3(3):e78.

12 Feehan LM, Geldman J, Sayre EC, Park C, Ezzat AM, Yoo JY, et al. Accuracy of Fitbit devices: systematic review and narrative syntheses of quantitative data. JMIR Mhealth Uhealth. 2018 Aug;6(8):e10527.

13 de Zambotti M, Goldstone A, Claudatos S, Colrain IM, Baker FC. A validation study of Fitbit Charge $2^{\mathrm{TM}}$ compared with polysomnography in adults. Chronobiol Int. 2018 Apr;35(4):465-76.

14 Shcherbina A, Mattsson CM, Waggott D, Salisbury H, Christle JW, Hastie T, et al. Accuracy in wrist-worn, sensor-based measurements of heart rate and energy expenditure in a diverse cohort. J Pers Med. 2017 May; 7(2):E3.

15 Pejovic V, Musolesi M. Anticipatory mobile computing: a survey of the state of the art and research challenges. ACM Comput Surv. 2015 Apr;47(3):47.

16 Reinertsen E, Clifford GD. A review of physiological and behavioral monitoring with digital sensors for neuropsychiatric illnesses. Physiol Meas. 2018 May;39(5):05TR01.

17 Doryab A, Chikarsel P, Liu X, Day AK. Extraction of Behavioral Features from Smartphone and Wearable Data. arXiv:1812.10394 [publication date 2018].

18 Chen R, Jankovic F, Marinsek N, Foschini L, Kourtis L, Signorini A, et al. Developing Measures of Cognitive Impairment in the Real World from Consumer-Grade Multimodal Sensor Streams. Proceedings of the 25th ACM SIGKDD International Conference on Knowledge Discovery \& Data Mining. ACM; 2019.

19 Foschini L, Medin J, Bezlyak V, Stuck D, Silva D, Lee WN. Real-World Use of Wearable Devices in a Large Multiple Sclerosis Cohort (P4.393). Neurology. 2018 Apr;90(15 Suppl):P4.393.

20 Samson SI, Lee WN, Quisel T, Foschini L, Liska J, Mills HG, et al. Using Claims and Consumer Wearable Devices Data to Quantify Influenza-Related Outcomes among Type 2 Diabetes Patients - A Large Population Study. Diabetes. 2018;67(Supplement 1):1616-P.

21 Coulman KD, Howes N, Hopkins J, Whale K, Chalmers K, Brookes S, et al.; By-Band-Sleeve Trial Management Group. A comparison of health professionals' and patients' views of the importance of outcomes of bariatric surgery. Obes Surg. 2016 Nov;26(11):2738-46.

22 Kim JJ, Rogers AM, Ballem N, Schirmer B; American Society for Metabolic and Bariatric Surgery Clinical Issues Committee. ASMBS updated position statement on insurance mandated preoperative weight loss requirements. Surg Obes Relat Dis. 2016 Jun;12(5):955-9.

23 Kuczmarski RJ, Ogden CL, Grummer-Strawn LM, Flegal KM, Guo SS, Wei R, et al. CDC growth charts: United States. Adv Data. 2000 Jun;(314):1-27. 